\title{
shRNA against CD44 inhibits cell proliferation, invasion and migration, and promotes apoptosis of colon carcinoma cells
}

\author{
YEON SUN PARK ${ }^{1}$, JUNG WOOK HUH ${ }^{1}$, JAE HYUK LEE $^{2}$ and HYEONG ROK KIM ${ }^{1}$ \\ Departments of ${ }^{1}$ Surgery, and ${ }^{2}$ Pathology, Chonnam National University Medical School \\ and Hwasun Hospital, Hwasun-gun, Gwangju 519-809, Republic of Korea
}

Received September 8, 2011; Accepted October 17, 2011

DOI: $10.3892 /$ or.2011.1532

\begin{abstract}
CD44 is a causal factor for tumor invasion, metastasis and acquisition of resistance to apoptosis. CD44 knockdown using inducible short hairpin RNA (shRNA) significantly reduces cell growth and invasion. Short hairpin RNA against CD44 and pGFP-V-RS-vector was used for knockdown of CD44 expression in SW620 colon cancer cells. Cell growth, invasion and migration assay, immunofluorescence for $\beta$-catenin expression and Western blotting for Wnt signaling molecules were analyzed. Cell cycle analysis and Western blot analysis for apoptotic molecules were evaluated. Short hairpin RNA against CD44 reduced the expression of CD44. Cell proliferation, migration and invasion were markedly inhibited and apoptosis was increased in shRNA CD44-transfected cells. Knockdown of CD44 decreased the phosphorylation of PDK1, Akt and GSK3 $\beta$, and $\beta$-catenin levels. Decreased phosphorylated Akt led to an increase in phosphorylated FoxO1 and induced cell cycle arrest in the $\mathrm{G}_{0}-\mathrm{G}_{1}$ phase and a decrease in the $\mathrm{S}$ phase. The levels of Bcl-2 and Bcl-xL expression were down-regulated, while the levels of BAX expression and cleaved caspase-3, -8 and -9 were increased. CD44 knockdown by way of shRNA inhibited cell proliferation and induced cell apoptosis. This can be used as a therapeutic intervention with the anti-survival/pro-apoptotic machinery in human colon cancer.
\end{abstract}

\section{Introduction}

Colorectal cancer (CRC) is one of the leading causes of cancerrelated deaths worldwide. Although a molecular genetic model of the preferential sequence has been proposed (1), the present knowledge of the cellular and molecular mechanisms of CRC can predict no biological parameter for the behavior of cancers. Normal and malignant cells display membrane receptors interacting with ligands present in the extracellular compartment

Correspondence to: Professor Hyeong Rok Kim, Department of Surgery, Chonnam National University Medical School, 160 Ilsimri, Hwasun-eup, Hwasun-gun, Gwangju 519-809, Republic of Korea E-mail:drkhr@jnu.ac.kr

Key words: CD44, short hairpin RNA, colon cancer of tissues. A prototypical example of this phenomenon is the hyaluronan receptor CD44 $(2,3)$. CD44 comprises a family of transmembrane glycoproteins generated from a single gene by alternative splicing and differential glycosylation $(4,5)$. Members of the CD44 family have been implicated in a number of important biological processes, including lymphocyte homing, apoptosis, tumor progression, and metastasis (6-9). In these processes, CD44 functions as a cell adhesion and a signaling receptor, linking the extracellular cytoskeleton (10). Furthermore, CD44 with heparin sulfate side chains can bind growth factors and modulate growth factor receptor-mediated signaling (11). Wnts are a highly conserved family of secreted growth factors that bind members of the frizzled family of transmembrane receptors, and through downstream signaling, proliferation, and differentiation $(12,13)$. The cytoplasmic/ nuclear pool of $\beta$-catenin involved in Wnt signaling is largely regulated by a multiprotein complex consisting of the APC tumor suppressor, AXIN, and GSK3 $\beta$ proteins (14-17). Nuclear localization of forkhead box transcription factor O (FoxO) proteins is required for transcriptional regulatory functions, which include the control of genes involved in apoptosis, such as Bim $(18,19)$ and FasL $(20)$, and genes involved in cell cycle regulation, such as p27 $(21,22)$ and cyclin D1 and D2 $(23,24)$. As major direct substrates of FoxO factors are negatively regulated by Akt phosphorylation in the presence of growth factor signaling $(25,26)$, which results in binding to $14-3-3$ proteins followed by nuclear export (27). Akt is recruited to the plasma membrane through the binding of its pleckstrin homology domain to the phosphatidylinositol 3,4,5-triphosphate (PIP3), which is a product of PI3K that is anchored to the plasma membrane. PDK1 is also recruited to the plasma membrane through interactions with PIP3. As both PDK1 and Akt interact with PIP3, PDK1 co-localizes with Akt and activates it by phosphorylating threonine 308 (T308-P) (28). Following its activation, Akt phosphorylates a number of downstream substrates which leads to the suppression of apoptosis, such as caspase-9 and glycogen synthase kinase $3 \beta$ (GSK-3 $\beta$ ) (29). The increased apoptosis might be associated with the ratios of Bcl-2 or Bcl-xL/Bax and activated caspase-3, -8 , and -9 $(30,31)$. CD44 and Fas are bound with preligand assembly domain blocking cell death signaling. Down-regulated CD44 makes Fas ligand free and induces increased cleaved form of caspases and apoptosis signaling (32). RNA interference (RNAi) is a post-transcriptional gene silencing mechanism 
Table I. Primers for RT-PCR.

\begin{tabular}{lll}
\hline Protein & Primers & Sequences \\
\hline CD44 & Forward & 5'-GAA TAT AAC CTG CCG CTT TG-3' \\
& Reverse & 5'-CTG AAG TGC TGC TCC TTT CAC-3' \\
GAPDH & Forward & 5'-ACC ACA GTC CAT GCC ATC AC-3' \\
& Reverse & 5'-TCC ACC ACC CTG TTG CTG TA-3' \\
\hline
\end{tabular}

which has demonstrated enormous prospects for human gene function, signal transduction research, and gene therapy. Specific gene silencing can be achieved in a variety of cell systems using chemically synthesized small interference RNA (siRNA). DNA vector-based short hairpin RNA (shRNA) is a sequence of RNA that makes a tight hairpin turn and can be used to silence gene expression via RNA interference $(33,34)$.

\section{Materials and methods}

Cell culture. SW620 colon carcinoma cells were obtained from the American Type Culture Collection (ATCC, Rockville, MD, USA). All cell lines were routinely maintained in complete medium (DMEM; Lonza, Switzerland) containing 10\% fetal bovine serum (Lonza), 100 units of penicillin/ml (Gibco ${ }^{\circledR}$ Invitrogen, San Diego, CA, USA), $100 \mu \mathrm{g}$ of streptomycin $/ \mathrm{ml}$ (Gibco Invitrogen). The cell lines were maintained at $37^{\circ} \mathrm{C}$ in $5 \% \mathrm{CO}_{2}$ and passed every 3-4 days.

shRNA CD44 transfection and selection. Short hairpin RNA with vector was purchased from OriGene (OriGene Technologies, Inc., Rockville, MD, USA). Short hairpin RNA-CD44 (sense: GACAGAAAG CCAAGTGGACTCAACGGAGA) and pGFP-V-RS-vector containing a non-effective shRNA cassette against GFP were used for knockdown of CD44 expression. Those individual clones were isolated and the stably-transfected cells were named as follows: SW620-S-CD44 (transfected with pGFP-V-RS shRNA-CD44) and SW620-NC [transfected with pGFP-V-RS-vector containing a non-effective shRNA cassette against GFP (negative)].

Reverse transcription-polymerase chain reaction. Total RNA was extracted from cultured cells using TRIzol reagent (Invitrogen). RNA (1 $\mu \mathrm{g} / \mu \mathrm{l})$ was used to synthesize cDNA using Superscript First-Strand Synthesis Kit (Promega, Madison, WI, USA) following the manufacturer's protocols. Expression of CD44 mRNA was detected with RT-PCR (Takara, Shiga, Japan) using specific primers (CD44 primer sense and antisense; Table I).

Western blotting. A total of $2.0 \times 10^{6}$ untransfected or stablytransfected cells were harvested by suspension in $50 \mu 1$ lysis buffer [50 mM Tris- $\mathrm{HCl}(\mathrm{pH} \mathrm{8.0)}$, Sigma]. Proteins were resolved by $10 \%$ SDS-PAGE and electro-blotted onto PVDF transfer membranes (GE Healthcare Life Sciences, Piscataway, NJ, USA), blocked with 5\% skim milk (Difco ${ }^{\circledR}$ BD PharMingen, San Jose, CA, USA) or $5 \%$ bovine serum albumin (BSA; Calbiochem, La Jolla, CA, USA), and probed with anti-CD44 (Santa Cruz Biotechnology, Inc., Santa Cruz, CA, USA), anti-PETN (Cell Signaling, Danvers, MA, USA), phospho-PDK1 (Cell Signaling), anti-AKT (Cell Signaling), anti-phospho-AKT (Ser/Thr; Cell Signaling), anti-GSK3 $\alpha / \beta$ (Cell Signaling), anti- $\beta$-catenin (Cell Signaling), anti-Bim (Cell Signaling), anti-Bcl-2 (Santa Cruz Biotechnology, Inc.), antiBcl-xL (Santa Cruz Biotechnology, Inc.), anti-Bax (Santa Cruz Biotechnology, Inc.), anti-Fas (Cell Signaling), anti-Fas-L (Cell Signaling), anti-phospho-p53 (Santa Cruz Biotechnology, Inc.), anti-p21 (Santa Cruz Biotechnology, Inc.), anti-p27 (Santa Cruz Biotechnology, Inc.), anti-cyclin D1 (Santa Cruz Biotechnology, Inc.), anti-CDK4 (Santa Cruz Biotechnology, Inc.), FoxO1 (AbFrontier, Seoul, Korea), anti-caspase 8, cleavaged-caspase 8 (Cell Signaling), anti-cleavaged-caspase 9 (Cell Signaling), anti-caspase 3, cleavaged-caspase 3 (Cell Signaling), or anti-GAPDH antibodies (Santa Cruz Biotechnology, Inc.). Following incubation with horseradish peroxidase-conjugated goat anti-mouse or anti-rabbit IgG (Sigma), the bands were visualized by chemiluminescence using a chemiluminescence kit (Millipore, Billerica, MA, USA). Immunoreactive bands were visualized on membranes using the Las-4000 system (Fujifilm Life Sciences, Piscataway, NJ, USA).

Growth curve. The untransfected or stably transfected cells were seeded in 96-well plates at a density of $1.0 \times 10^{4}$ cells/well in DMEM containing 10\% fetal bovine serum (FBS; Lonza) at a final volume of $0.2 \mathrm{ml}$, and grown for 15 days at $37^{\circ} \mathrm{C}$ with $5 \% \mathrm{CO}_{2}$. During this period, we selected three wells from each group of cells every day at random for the growth curve assay.

Colony formation assay. Approximately $2.0 \times 10^{2}$ SW620 cells untransfected or stably-transfected with SW620-S-CD44 and SW620-NC vectors were plated in 60-mm culture dishes (SPL, Gyeonggi-Do, Korea). After 15 days, the cells were fixed with methanol and stained with $0.1 \%$ crystal violet (Sigma). Visible colonies were manually counted and their size was measured.

Cell migration assay. Cell migration was evaluated by a scratch wound-healing assay. Cells $\left(5 \times 10^{5}\right.$ per well) were seeded in the culture insert (Ibidi ${ }^{\odot} \mathrm{GmbH}$, Martinsried, Germany) and allowed to adhere for $24 \mathrm{~h}$. After appropriate cell attachment, the culture insert was gently removed using sterile tweezers. Fresh, full medium was added and the cells were allowed to close the wound for $48 \mathrm{~h}$. Photographs were taken every $24 \mathrm{~h}$ at the same position of the wound and the migration distance was measured.

Cell invasion assay. The cell invasion assay was performed using a two-chamber Transwell (SPL). The upper surface of a polycarbonate filter with $8-\mu \mathrm{m}$ pores was coated with $1 \mathrm{mg} / \mathrm{ml}$ of Matrigel (BD PharMingen). Cells (2.5x10 $)$ were suspended in DMEM (Lonza) supplemented with FBS and added to the upper chamber, and serum-free DMEM media was placed in the lower chamber. Cells were incubated for $24 \mathrm{~h}$ at $37^{\circ} \mathrm{C}$ in a $5 \% \mathrm{CO}_{2}$ incubator. At the end of incubation, the cells on the upper surface of the filter were completely removed by wiping them with a cotton swab. Then, the filters were fixed in $75 \%$ ethanol and stained with hematoxylin and 
Table II. DNA sequences of Wnt1a/Wnt3a.

\begin{tabular}{ll}
\hline Wnt & DNA sequence \\
\hline Wnt1a & CGCGCGTCTGCACGAGTGTCTATCCATGGCCTACCC \\
Wnt3a & CGTGCACACCTGCAAGTCCATGGCCTACCC \\
\hline
\end{tabular}

eosin (Millipore). Cells that had invaded the Matrigel and reached the lower surface of the filter were counted under a light microscope.

Immunofluorescence for $\beta$-catenin expression. Prosta-sphere images were analyzed for expression of nuclear $\beta$-catenin following untransfected or shRNA CD44-transfected cells. Confocal imaging was performed using a Zeiss LSM 510 with a $x 4$ objective zoom 2-3. Images were analyzed using personal computer-based FV10-ASW 1.7 software (Olympus, Tokyo, Japan). DAPI nuclear staining (blue; Invitrogen) and quantified the intensities of the pixels in each channel (DAPI, blue; $\beta$-catenin, red; Alexa Fluor ${ }^{\circledR} 594$ conjugate; Cell Signaling) on the sections were analyzed and histograms were generated.

PI3K inhibitor. SW620-NC and SW620-S-CD44 cells were cultured as described above. Cells $\left(2.0 \times 10^{6}\right)$ were plated in DMEM containing 10\% FBS. Twenty-four hours after the cells were seeded in a 10-cm culture dish, $20 \mu \mathrm{m}$ of LY294002 (Cell Signaling) was added to the serum-free medium for $24 \mathrm{~h}$. Cells were washed in PBS and lysed and used in Western blot analysis as described above.
Wnt la/3a transfection with pLNCX retroviral vector. The primer sequences of sense cDNA of Wnt 1a and 3a are shown in Table II and cloned in the retroviral vector, pLNCX (Clontech, Mountain View, CA, USA). When the cells reached 60-80\% confluence, each cell was transfected with pLNCX-Wnt 1a/ pLNCX-Wnt 3a using Lipofectamine 2000 (Invitrogen). At $48 \mathrm{~h}$ post-transfection, cells were harvested and lysed for Western blotting.

Flow cytometry. Cells were stained with allophycocyanin (APC)-labeled Annexin-V and 7-amino-actinomycin D (7-AAD, Annexin-V Apoptosis kit; Abcam, Cambridge, UK). We classified the cells as follows: intact cells (Annexin $/$ 7-AAD ${ }^{-}$); apoptotic cells (Annexin ${ }^{+} / 7-\mathrm{AAD}^{-}$); and necrotic cells $\left(\right.$ Annexin ${ }^{+} / 7-\mathrm{AAD}^{+}$). A total of $5.0 \times 10^{5}$ untransfected or stably-transfected SW620 cells were washed twice with ice-cold PBS and incubated for $5 \mathrm{~min}$ in a binding buffer $(1 \mu \mathrm{g} / \mathrm{ml}$ of $7-A A D$ and $1 \mu \mathrm{g} / \mathrm{ml}$ of APC-labeled Annexin-V). FACS analysis for Annexin-V and 7-AAD stained cells was performed. Cell cycle analysis was performed with Hoechst 33258 using the bromodeoxyuridin (brdUrd) Flow kit (BD PharMingen).

Statistical analysis. All statistical analyses were performed using a t-test with SPSS 17.0 (SPSS Inc., Chicago, IL, USA). All data are expressed as the mean \pm SD.

\section{Results}

Expression of CD44 $\mathrm{mRNA}$ and protein. The level of expression of CD44 mRNA was evaluated by RT-PCR; GAPDH served as an internal control. As shown in Fig. 1A, the level
A
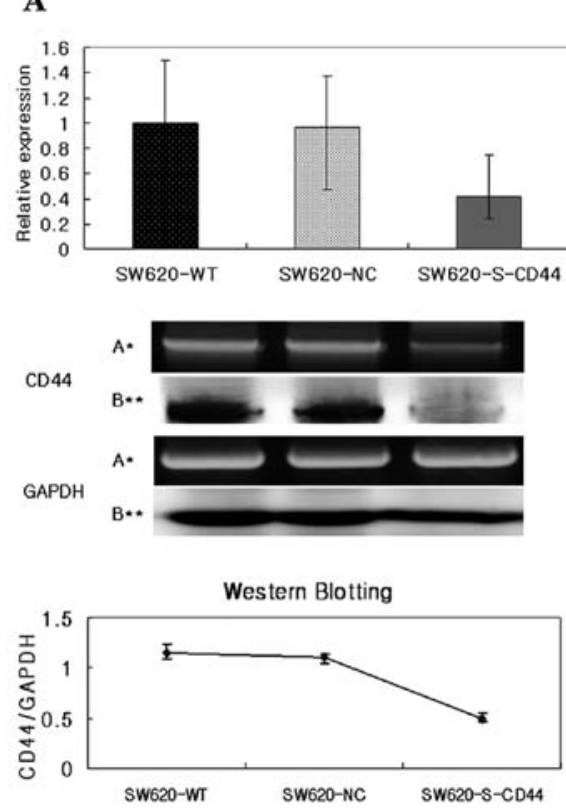

B
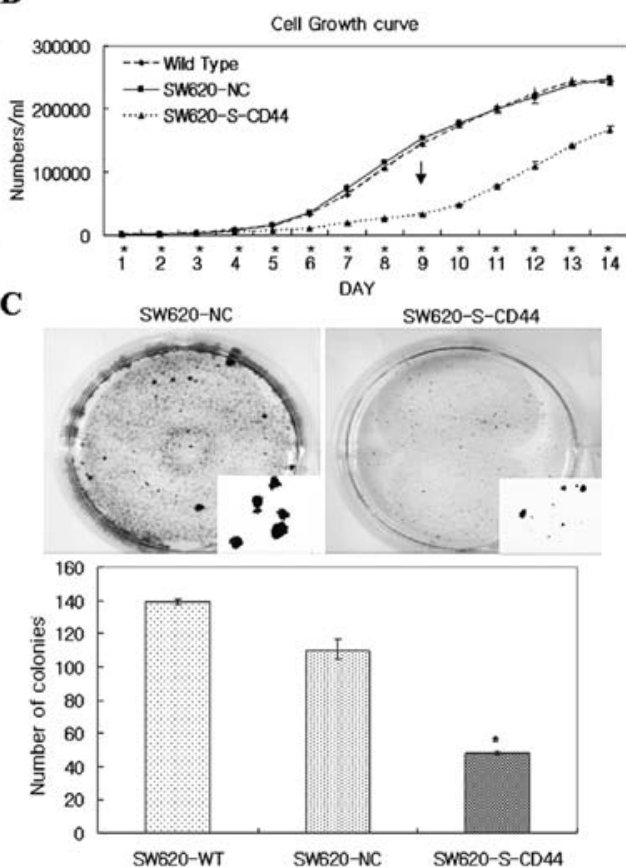

Figure 1. CD44 expression and cell proliferation. (A) mRNA expression of SW620-S-CD44 was reduced by 57.36\% (p<0.05). The protein expression of SW620-S-CD44 was reduced by 57.23\% (p<0.05). (A*, RT-PCR; B**, Western blot analysis). (B) The proliferation of SW620-S-CD44 was markedly inhibited in a time-dependent manner, and the highest inhibition rate was $61.92 \pm 2.4 \%$ on day $9(\mathrm{p}<0.05)$. (C) The number of colonies in SW620-S-CD44 was decreased with an inhibition rate of $56.36 \%(\mathrm{p}<0.005)$. 
A
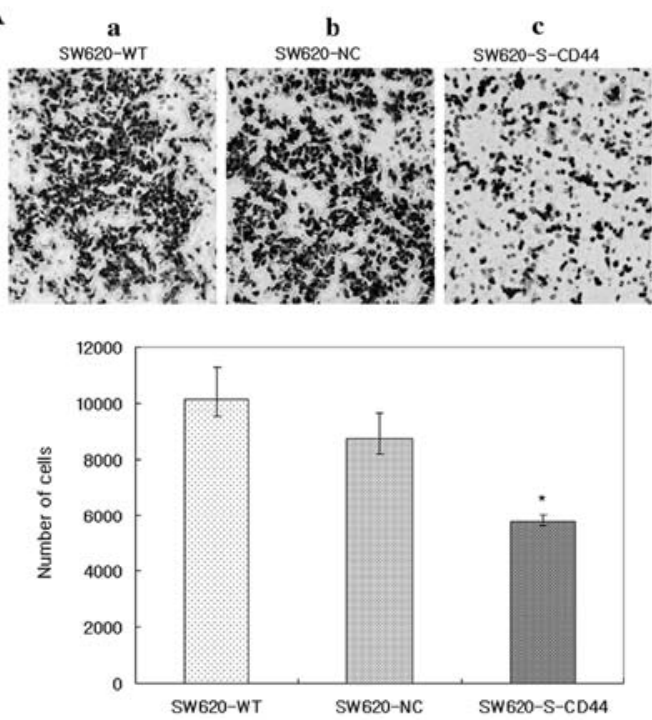

B
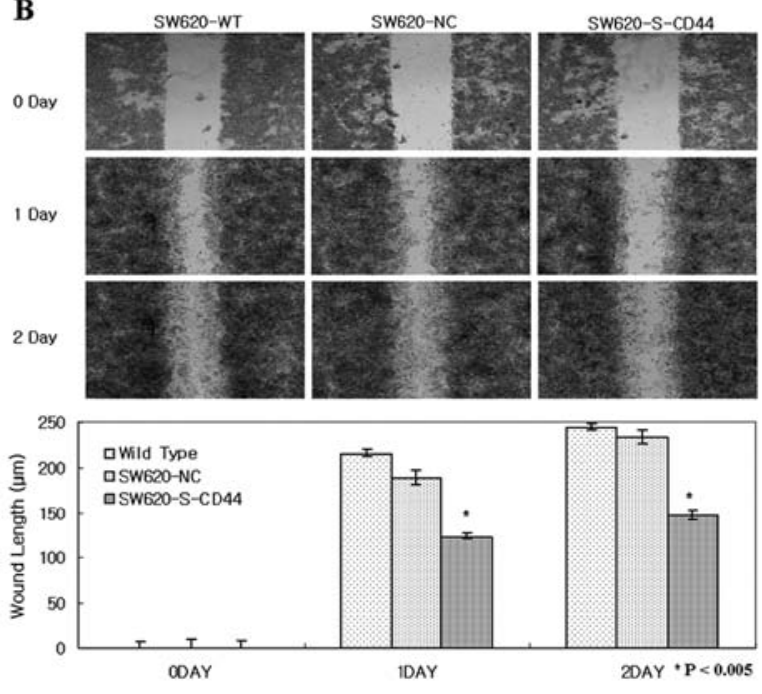

Figure 2. Invasion and migration assay. (A) The cells that invaded through the Matrigel-coated inserts were counted and photographed under a light microscope (x400). a, SW620-WT; b, SW620-NC; c, SW620-S-CD44. The invasion activity of SW620-S-CD44 cells was significantly decreased by 43.4\% compared to SW620-WT ( ${ }^{*}$ p $\left.<0.001\right)$. (B) The migration assay was evaluated by the distance $(\mu \mathrm{m})$ between the foremost-migrated cell and start line. The image was photographed under a light microscope (x100) in 24 and $48 \mathrm{~h}$. The migration activity of SW620-S-CD44 cells was significantly decreased by $42.69 \%$ (24 h) and $40.35 \%(48 \mathrm{~h})$ compared to SW620-WT ( $\left.{ }^{*} \mathrm{p}<0.005\right)$.
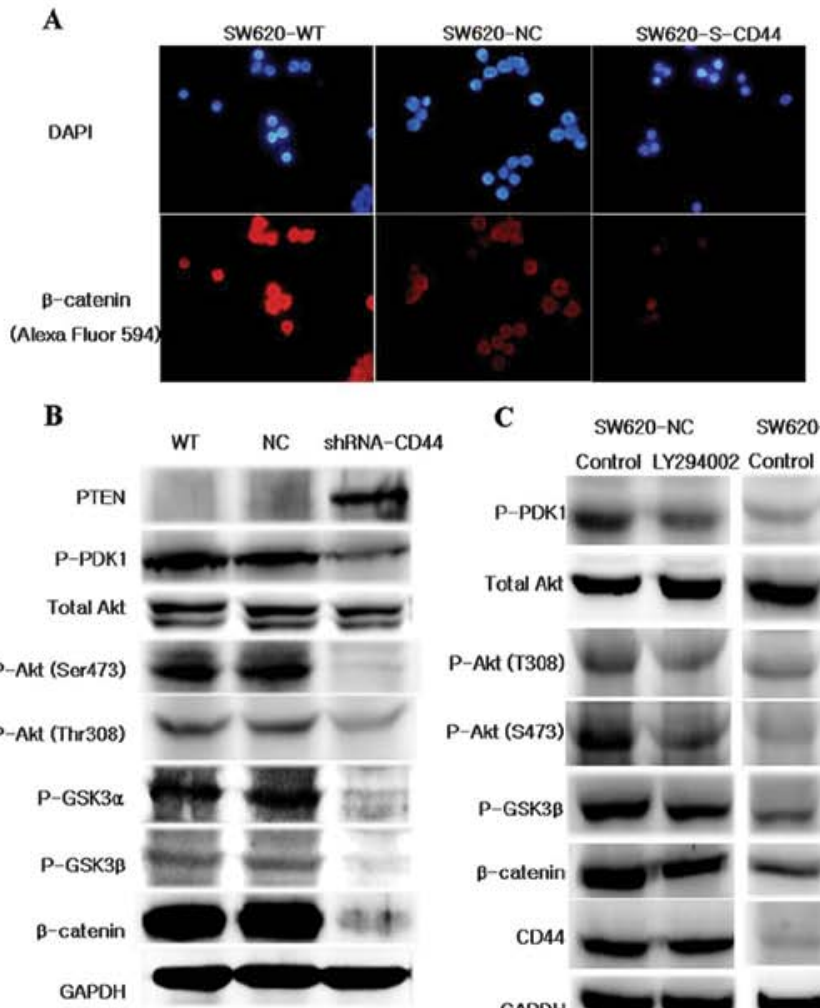

C

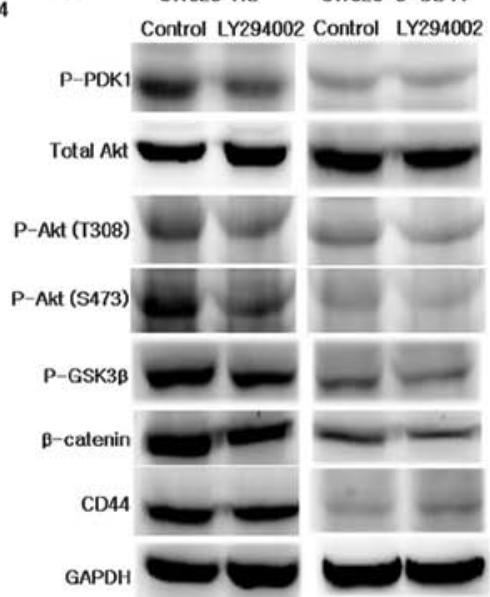

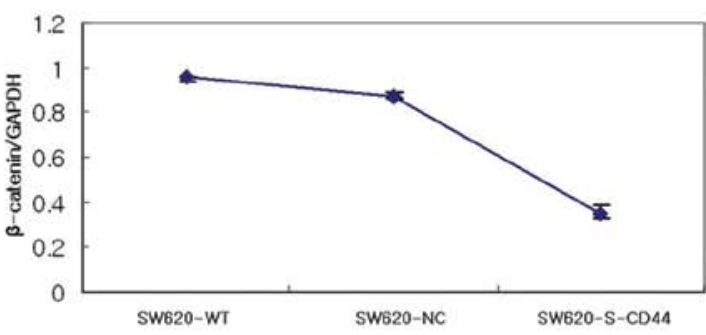

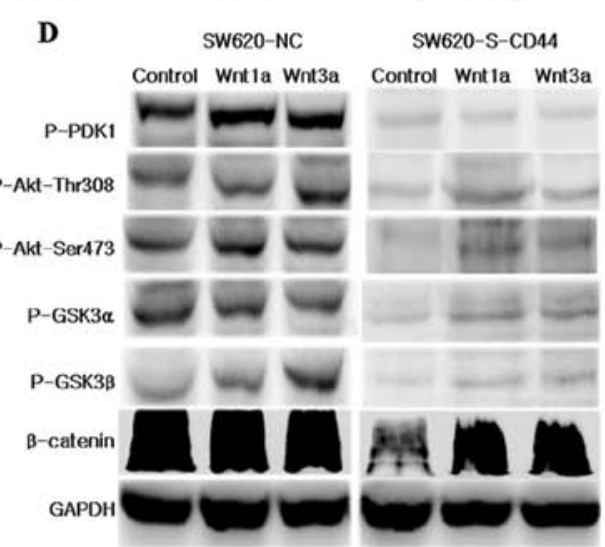

Figure 3. $\beta$-catenin expression and Wnt signaling pathway. (A) Immunofluorescence microscopy images of the sub-cellular localization of $\beta$-catenin. A red signal indicates $\beta$-catenin. The $\beta$-catenin activity of SW620-S-CD44 was significantly decreased by $56.4 \%$ compared to wild-type ("p<0.001). (B) Western blot analysis of SW620-S-CD44 showed decreased phosphorylation of AKT, phosphorylation of GSK3 $\alpha / \beta$, and phosphorylation of PDK1 (Ser241). (C) LY294002 treatment significantly inhibited phosphorylated Akt expression and $\beta$-catenin in SW620-NC, but SW620-S-CD44. (D) After Wnt1a/3a transfection into SW620-S-CD44, the expression of phospho Akt, GSK3, and $\beta$-catenin were recovered.

of expression of CD44 mRNA in SW620-S-CD44 cells was significantly down-regulated $(\mathrm{p}<0.05)$. A significant reduction in CD44 protein expression was detected in shRNA CD44transfected cells (SW620-S-CD44) compared with parental 


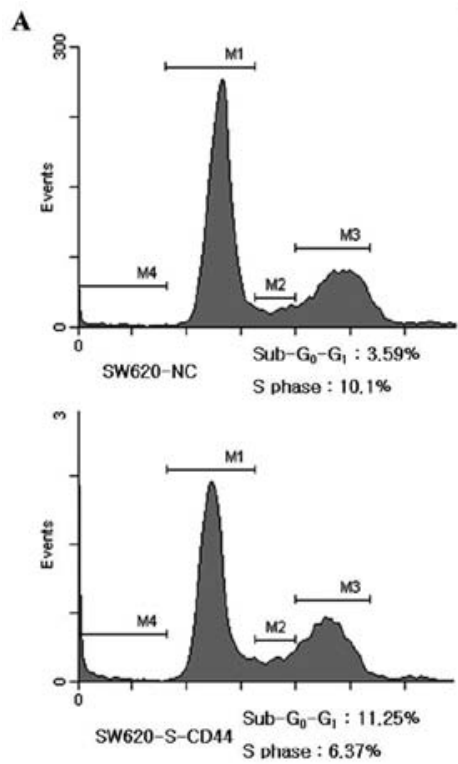

C

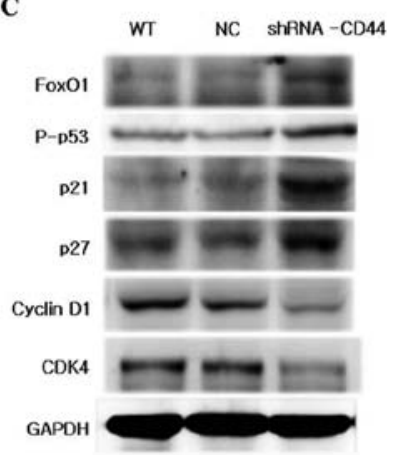

B
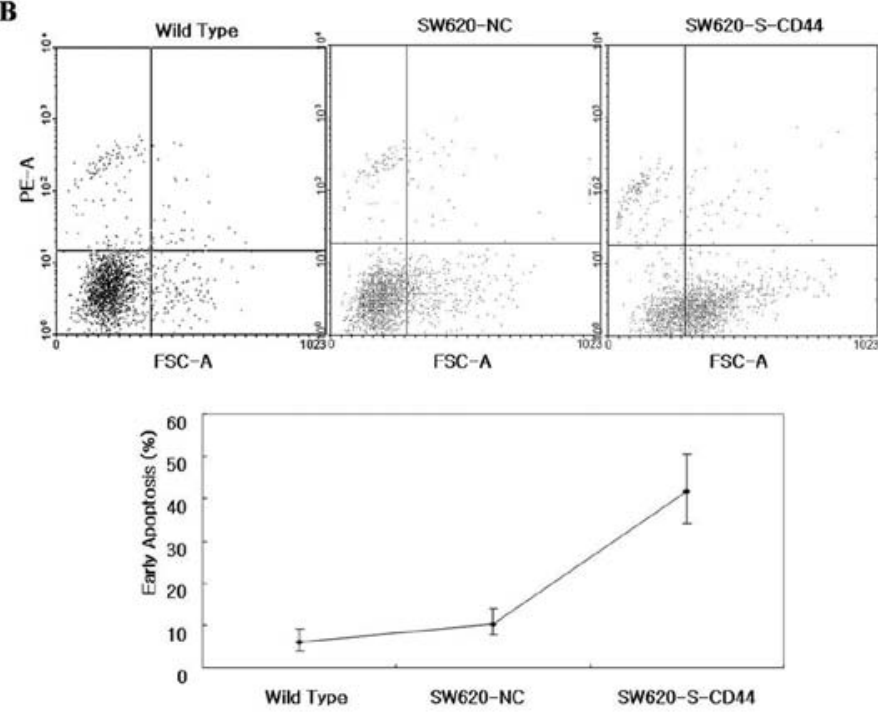

D

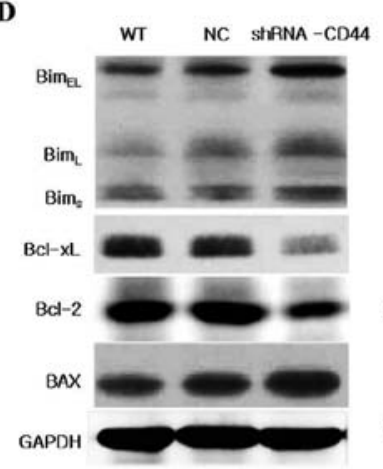

E

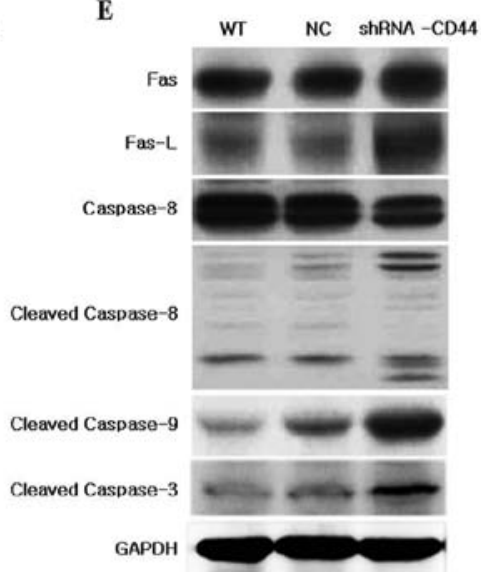

Figure 4. Flow cytometry and Western blot analysis for apoptotic molecules. (A) Flow cytometry reveals increase in the sub- $\mathrm{G}_{0}-\mathrm{G}_{1}$ phase and decrease in the $\mathrm{S}$ phase in SW620-S-CD44 compared to SW620-NC (p<0.001). (B) The early apoptosis rate of SW620-S-CD44 was significantly increased compared to control cells ("p<0.005). (C) SW620-S-CD44 increased the levels of FoxO1, p53, p21, p27, and decreased cyclin D1 and CDK4 (D) SW620-S-CD44 decreased the

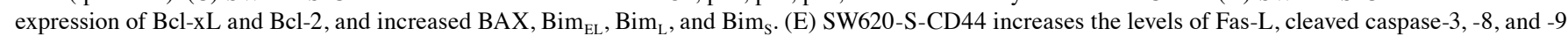
and decreases the levels of uncleaved caspase-8.

(SW620-WT) and non-effective shRNA-transfected cells (SW620-NC; p<0.05).

Cell growth and colony formation. Growth assay was performed to examine the cell proliferation activity with SW620-S-CD44, SW620-NC, and SW620-WT. As shown in Fig. 1B, the cell proliferation was significantly decreased in shRNA-transfected cells compared with control cells, and the inhibition rate on day 9 was $61.92 \pm 2.4 \%(\mathrm{p}<0.05)$. SW620-S-CD44 stable cells show a significant reduction of colony number $(\mathrm{p}<0.005$, Fig. $1 \mathrm{C})$. In addition, the size of SW620-S-CD44 colonies was smaller than SW620-WT and SW620-NC (Fig. 1C).

Invasion and migration assay. SW620-S-CD44 showed much lower invasion activities than other control cells (SW620-NC and SW620-WT) and the invasion activity was decreased by approximately $43.4 \%$ (Fig. 2A). Migration was significantly decreased in shRNA-transfected cells compared with the other control cells, and the highest inhibition rate (SW620S-CD44/SW620-WT) was $42.69 \%$ (24 h), 40.35\% (48 h; p<0.005; Fig. 2B). SW620-S-CD44 took $>48$ h to close a wound, whereas SW620-WT and SW620-NC cells took $<48 \mathrm{~h}$ to heal a wound.

$\beta$-catenin activities and PDK1/Akt/GSK3 phosphorylation. Transfected shRNA-CD44 led to down-regulation of the $\beta$-catenin level (Fig. 3A and B) based on an immunofluorescence assay. In terms of the $\mathrm{Wnt} / \beta$-catenin signaling pathway, Akt activation requires phosphorylation of the Thr308 site by PDK1. As shown in Fig. 3B, shRNA CD44 inhibited PDK1 phosphorylation and caused a decrease in the intensity of the phosphorylated Akt. shRNA CD44 also inhibited GSK3 $\alpha / \beta$ phosphorylation.

PI3k inhibitor. LY294002 blocks H2-relaxin-induced phosphorylation of Akt and GSK-3 $\beta$. We used the PI3K inhibitor, LY294002, to block PI3K/Akt activation. LY294002 treatment 
significantly inhibited phosphorylated Akt expression and $\beta$-catenin (Fig. 3C). Complete inhibition of Akt phosphorylation at Thr308 and Ser473 was observed, while the total amount of Akt proteins remained the same in the presence or absence of LY294002. Consistent with the decrease in $\beta$-catenin protein levels, we found that Ser9 phosphorylation of GSK-3 $\beta$ proteins was also impaired by treatment with LY294002. However, there was minimal or no change of phosphorylated Akt expression in the SW620-S-CD44.

Wntla/3a transfection. To confirm inhibition of Wnt signaling by shRNA CD44, Wntla/3a sequences were transfected into SW620-S-CD44. After transfection of Wnt 1a/3a, PDK1/Akt, GSK3 $\alpha / \beta$, and $\beta$-catenin levels recovered (Fig. 3D).

Cell cycle analysis. Cell cycle analysis showed that the $\mathrm{G}_{0}-\mathrm{G}_{1}$ population was increased from $2 \%$ in SW620-NC to $11.25 \%$ in SW620-S-CD44 and the S-phase fraction was decreased from 10.1 to $6.37 \%$ (Fig. 4A; p<0.001). Western blot analysis showed increased levels of phosphorylated p53 and increased the transcription of FoxO1 and p53. SW620-S-CD44 also increased the expression of p21 and p27, and decreased cyclin D1 and CDK 4 , the activity of which is required for the cell cycle $G_{1} / S$ transition (Fig. 4C).

Apoptosis analysis. The apoptotic rate of SW620-S-CD44 was increased to $41.7 \%$ compared with $10.3 \%$ in SW620-NC based on FACS analysis ( $p<0.005$; Fig. 4B). shRNA CD44 decreased the expression of $\mathrm{Bcl}-\mathrm{xL}$ and $\mathrm{Bcl}-2$ proteins, but increased the expression of $\mathrm{BAX}, \mathrm{Bim}_{\mathrm{EL}}, \mathrm{Bim}_{\mathrm{L}}$ and $\mathrm{Bim}_{\mathrm{S}}$ (Fig. 4D). shRNA CD44 also increased the expression of Fas-L, cleaved caspase-3, -8, and -9 (Fig. 4E).

\section{Discussion}

CD44 is a transmembrane cell-surface adhesion molecule that has been found to support anchorage-independent growth in vitro and tumor growth and metastasis in experimental models of solid cancers (6-8). The CD44 is involved in fundamental aspects of cancer cell biology, such as the tumor stem cell phenotype, cell adhesion, and invasion, and this inhibition provides a rationale for the design of corresponding therapies $(8,35)$. RNA interference can be triggered by small interfering RNAs (siRNAs) that cause inhibition of gene expression on specific genes (36). These siRNAs can be used for the suppression of gene expression. Similarly, shRNA is a DNA vector-based shRNA which can be further processed to form siRNAs. The shRNA hairpin structure is cleaved by the cellular machinery into siRNA (37). Proliferation and invasion of tumor cells is under the control of several different signaling pathways, including Wnt signaling (15). Aberrant Wnt signaling initiates malignant transformation of intestinal epithelium in both humans and mice (38), perturbing the proliferation, migration, differentiation, and apoptosis of progenitor cells in colon cancer (39). In the present study, CD44 is an important regulator of cell adhesion, tumor initiation, migration, and proliferation targeting Wnt signaling. In tumors with Wnt pathway defects, stabilized $\beta$-catenin interacts with TCF transcription factors to mediate increased expression of specific genes, at least some of which likely play critical roles in cancer pathogenesis (40). One of the well-known pathways that regulates oncogenesis in different organs is the Wnt signaling pathway (41). $\beta$-catenin is a downstream molecule in the Wnt signaling pathway and plays important roles in the structural organization and function of cell-to-cell adhesion and tumor invasion and metastasis (42). CD44 can also regulate Wnt-target genes (43). Among them, Akt is important for the survival of cancer cells (21). The Akt phosphorylation was inhibited by shRNA CD44 and PDK1, which controls the activation of Akt. The site of PDK1 phosphorylation, Ser 241, is very important in PDK1 activation $(44,45)$. shRNA CD44 inhibited Ser241 phosphorylation of PDK1. This inhibition of the PDK1/Akt molecule is an important signal for cell survival (27). GSK $3 \beta$ is the target of PDK1/Akt signal transduction. Down-regulated GSK3 $\beta$ inactivates various proteins that are involved in cell proliferation and survival, such as $\beta$-catenin, cyclin D1, c-jun, c-myc, C/EBP and CREB (46). The loss of GSK3 protein explains the stable accumulation of nonphosphorylated $\beta$-catenin that is accessible to the adherens junction at the cell periphery (47). The Wnt/ $\beta$-catenin pathway was very recently reported to protect cells from $\mathrm{p} 53$-mediated FoxO1-induced apoptosis, a mechanism involving the activation of the Akt survival pathway $(27,29)$. Silencing of CD44 expression in SW620 cells, indicating decreased $\beta$-catenin, increased wild-type p53 and FoxO1 expression. Activation of FoxO1 induces apoptosis by up-regulating a number of cell death genes, including those encoding the ligand for the death receptor (Fas) and the Bcl-2-interacting mediator (Bim) of cell death, and the tumor necrosis factor-related apoptosis-inducing ligand (27,33). Sub-cellular localization of wild-type FoxO1 is controlled primarily by phosphorylation, leading to nuclear export and subsequent repression of transcriptional activity $(25,26)$. BAX is one of the pro-apoptotic member proteins, and is activated by the FoxO1 and Bcl-family (Bcl-2 and Bcl-xL). BAX induces apoptosis due to a caspase-mediated mitochondrial pathway. As CD44 offers most resistance to apoptosis in human colon cancer cells, CD44 knockdown cells exhibit increased FoxO1, BAX, and cleaved caspase levels. These results suggest that the increased apoptosis in the CD44 knockdown group could be due to reduced clonogenic ability (19,20,48-50). The Bcl-2 family of proteins performs antiapoptotic roles in the regulation of mitochondrial membrane permeability (33). The knockdown of CD44 was shown to alter the levels of Bcl-2 family member proteins. The levels of anti-apoptotic Bcl-xL/Bcl-2 and Bid proteins were decreased and pro-apoptotic BAX and Bim levels were increased in shRNA CD44-transfected cells.

Caspases are a family of proteases that are collectively known as one of the principal executioners of apoptosis, and their cleavage and subsequent activation are considered the primary hallmarks of apoptosis. In the death receptordependent pathway, death receptors and their ligands (Fas/ Fas-L and DR5/TRAIL) induce caspase- 8 activation; activated caspase- 8 in turn cleaves Bid and/or directly triggers the activation of caspase-3. When cleaved, truncated Bid migrates to the mitochondria, where it increases the permeability of the mitochondrial membrane, induces cytochrome c release and caspase- 9 cleavage (31). The current study demonstrated that shRNA CD44 induces apoptosis in SW620 human colon cancer cells harboring the wild-type p53 gene, and inducing the 
cleavage of caspase-3, -8 , and -9. In conclusion, knockdown of CD44 by way of shRNA inhibits cell proliferation and invasion by down-regulation of the Wnt/ $\beta$-catenin/PDK1/Akt signaling and induces cell apoptosis by increased $G_{0}-G_{1}$ phase/activation of caspase activity. This suggests that CD44 knockdown can be a therapeutic intervention with anti-survival/pro-apoptotic machinery in human colon cancer.

\section{Acknowledgements}

This study was supported by a grant from Chonnam National University 2010, Research Institute of Medical Sciences, Chonnam National University (2011-CURIMS-DR007) and a Research Grant 0720570 from the National Cancer Center, South Korea.

\section{References}

1. Grady WM and Carethers JM: Genomic and epigenetic instability in colorectal cancer pathogenesis. Gastroenterology 135: 1079-1099, 2008.

2. Cichy J and Pure E: The liberation of CD44: J Cell Biol 161: 839-843, 2003

3 Nakamura $\mathrm{H}$, Suenaga N, Taniwaki, et al: Constitutive and induced CD44 shedding by ADAM-like proteases and membrane-type 1 matrix metalloproteinase. Cancer Res 64 876-882, 2004.

4. Bourguignon LY: CD44-mediated oncogenic signaling and cytoskeleton activation during mammary tumor progression. J Mammary Gland Biol Neoplasia 6: 287-297, 2001.

5. Gotte M and Yip GW: Heparanase, hyaluronan, and CD44 in cancers: a breast carcinoma perspective. Cancer Res 66 : 10233-10237, 2006.

6. Kurozumi K, Nakao K, Nishida T, et al: Significance of biologic aggressiveness and proliferating activity in papillary thyroid carcinoma. World J Surg 22: 1237-1242, 1998.

7. Bankfalvi A, Terpe HJ,Breukelmann D, et al: Immunophenotypic and prognostic analysis of E-cadherin and beta-catenin expression during breast carcinogenesis and tumour progression: a comparative study with CD44. Histopathology 34: 25-34, 1999.

8. Takahashi K, Stamenkovic I, Cutler M, et al: CD44 hyaluronate binding influences growth kinetics and tumorigenicity of human colon carcinomas. Oncogene 11: 2223-2232, 1995.

9. Shtivelman E and Bishop JM: Expression of CD44 is repressed in neuroblastoma cells. Mol Cell Biol 11: 5446-5453, 1991.

10. Ponta H, Sherman L and Herrlich PA: CD44: from adhesion molecules to signalling regulators. Nat Rev Mol Cell Biol 4: 33-45, 2003.

11. Johnson P, Maiti A, Brown KL and Li R: A role for the cell adhesion molecule CD44 and sulfation in leukocyte-endothelial cell adhesion during an inflammatory response? Biochem Pharmacol 59: 455-465, 2000.

12. Liu S, Dontu G, Mantle ID, et al: Hedgehog signaling and Bmi-1 regulate self-renewal of normal and malignant human mammary stem cells. Cancer Res 66: 6063-6071, 2006.

13. Lowry WE, Blanpain C, Nowak JA, et al: Defining the impact of beta-catenin/Tcf transactivation on epithelial stem cells. Genes Dev 19: 1596-1611, 2005

14. Reynolds $\mathrm{AB}$ and Roczniak-Ferguson $\mathrm{A}$ : Emerging roles for p120-catenin in cell adhesion and cancer. Oncogene 23 : 7947-7956, 2004

15. Logan CY and Nusse R: The Wnt signaling pathway in development and disease. Annu Rev Cell Dev Biol 20: 781-810, 2004.

16. Iba T, Kigawa J, Kanamori Y, et al: Expression of the c-myc gene as a predictor of chemotherapy response and a prognostic factor in patients with ovarian cancer. Cancer Sci 95: 418-423, 2004.

17. Peifer M: Beta-catenin as oncogene: the smoking gun. Science 275: 1752-1753, 1997.

18. Gilley J, Coffer PJ and Ham J: FOXO transcription factors directly activate bim gene expression and promote apoptosis in sympathetic neurons. J Cell Biol 162: 613-622, 2003.
19. Dijkers PF, Medema RH, Lammers JW, et al: Expression of the pro-apoptotic Bcl-2 family member Bim is regulated by the forkhead transcription factor FKHR-L1. Curr Biol 10: 1201-1204, 2000.

20. Brunet A, Bonni A, Zigmond MJ et al: Akt promotes cell survival by phosphorylating and inhibiting a Forkhead transcription factor. Cell 96: 857-868, 1999.

21. Medema RH, Kops GJ, Bos JL and Burgering BM: AFX-like Forkhead transcription factors mediate cell-cycle regulation by Ras and PKB through p27kip1. Nature 404: 782-787, 2000.

22. Dijkers PF, Medema RH, Pals C, et al: Forkhead transcription factor FKHR-L1 modulates cytokine-dependent transcriptional regulation of p27(KIP1). Mol Cell Biol 20: 9138-9148, 2000.

23. Ramaswamy S, Nakamura N, Sansal I, et al: A novel mechanism of gene regulation and tumor suppression by the transcription factor FKHR. Cancer Cell 2: 81-91, 2002.

24. Schmidt M, Fernandez de Mattos S, van der Horst A, et al: Cell cycle inhibition by FoxO forkhead transcription factors involves downregulation of cyclin D. Mol Cell Biol 22: 7842-7852, 2002.

25. Greer E and Brunet A: FOXO transcription factors at the interface between longevity and tumor suppression. Oncogene 24: 7410-7425, 2005.

26. Brownawell AM, Kops GJ, Macara IG and Burgering BM: Inhibition of nuclear import by protein kinase $\mathrm{B}(\mathrm{Akt})$ regulates the subcellular distribution and activity of the forkhead transcription factor AFX. Mol Cell Biol 21: 3534-3546, 2001.

27. Zhao X, Gan L, Pan H, et al: Multiple elements regulate nuclear/ cytoplasmic shuttling of FOXO1: characterization of phosphorylation- and 14-3-3-dependent and -independent mechanisms. Biochem J 378: 839-849, 2004.

28. Alessi DR, Andjelkovic M, Caudwell B, et al: Mechanism of activation of protein kinase B by insulin and IGF-1. EMBO J 15: 6541-6551, 1996

29. Sarbassov DD, Guertin DA, Ali SM and Sabatini DM: Phosphorylation and regulation of Akt/PKB by the rictor-mTOR complex. Science 307: 1098-1101, 2005.

30. Farrow SN and Brown R: New members of the Bcl-2 family and their protein partners. Curr Opin Genet Dev 6: 45-49, 1996.

31. Pathan N, Marusawa H, Krajewska, et al: TUCAN, an antiapoptotic caspase-associated recruitment domain family protein overexpressed in cancer. J Biol Chem 276: 32220-32229, 2001.

32. Mielgo A, van Driel M, Bloem A, et al: A novel antiapoptotic mechanism based on interference of Fas signaling by CD44 variant isoforms. Cell Death Differ 13: 465-477, 2006.

33. Rui W, Xi W, Fang L, et al: shRNA-targeted cyclooxygenase (COX)-2 inhibits proliferation, reduces invasion and enhances chemosensitivity in laryngeal carcinoma cells. Mol Cell Biochem 317: 179-188, 2008.

34. Suniti M, Vincent CH, Carla DG, et al: Delivery of CD44 shRNA/Naboparticles within cancer cells. J Biol Chem 284: 12432-12446, 2009.

35. Weber GF, Bronson RT, Ilagan J, et al: Absence of the CD44 gene prevents sarcoma metastasis. Cancer Res 62: 2281-2286, 2002.

36. Elbashir SM, Harborth J, Lendeckel W, et al: Duplexes of 21-nucleotide RNAs mediate RNA interference in cultured mammalian cells. Nature 411: 494-498, 2002.

37. Paddison PJ, Caudy AA, Bernstein E, et al: Short hairpin RNAs (shRNAs) induce sequence-specific silencing in mammalian cells. Genes Dev 16: 948-958, 2002.

38. Clevers $\mathrm{H}$ : Wnt/beta-catenin signaling in development and disease. Cell 127: 469-480, 2006.

39. Hall CL and Keller ET: The role of Wnts in bone metastases. Cancer Metastasis Rev 25: 551-558, 2006.

40. Tetsu $\mathrm{O}$ and McCormick F: Beta-catenin regulates expression of cyclin D1 in colon carcinoma cells. Nature 398: 422-426, 1999.

41. Hall CL, Bafico A, Dai J, et al: Prostate cancer cells promote osteoblastic bone metastases through Wnts. Cancer Res 65: 7554-7560, 2005.

42. Mao B, Wu W, Davidson G, et al: Kremen proteins are Dickkopf receptors that regulate Wnt/beta-catenin signalling. Nature 417: 664-667, 2002.

43. Ishimoto T, Oshima $\mathrm{H}$, Oshima M, et al: $\mathrm{CD} 44^{+}$slow-cycling tumor cell expansion is triggered by cooperative actions of Wnt and prostaglandin E2 in gastric tumorigenesis. Cancer Sci 101: 673-678, 2010 
44. Furumoto Y, Brooks S, Olivera A, et al: Cutting Edge: lentiviral short hairpin RNA silencing of PTEN in human mast cells reveals constitutive signals that promote cytokine secretion and cell survival. J Immunol 176: 5167-5171, 2006.

45. Wick MJ, Dong LQ, Riojas RA, et al: Mechanism of phosphorylation of protein kinase B/Akt by a constitutively active 3-phosphoinositide-dependent protein kinase-1. J Biol Chem 275: 40400-40406, 2000

46. Downward J: PI 3-kinase, Akt and cell survival. Semin Cell Dev Biol 15: 177-182, 2004

47. Casamayor A, Morrice NA and Alessi DR: Phosphorylation of Ser-241 is essential for the activity of 3-phosphoinositidedependent protein kinase-1: identification of five sites of phosphorylation in vivo. Biochem J 342 (Pt 2): 287-292, 1999.
48. Sorenson CM, Padanilam BJ and Hammerman MR: Abnormal postpartum renal development and cystogenesis in the bcl-2 (-/-) mouse. Am J Physiol 271: F184-F193, 1996.

49. Sorenson CM, Rogers SA, Korsmeyer SJ and Hammerman MR: Fulminant metanephric apoptosis and abnormal kidney development in bcl-2-deficient mice. Am J Physiol 268: F73-F81, 1995.

50. Chandler D, el-Naggar AK, Brisbay S, et al: Apoptosis and expression of the bcl-2 proto-oncogene in the fetal and adult human kidney: evidence for the contribution of bcl-2 expression to renal carcinogenesis. Hum Pathol 25: 789-796, 1994. 